\title{
Vacuum sealing drainage combined with free anterolateral femoral skin flap grafting in 16 cases of pediatric soft tissue damage to the foot and ankle
}

\author{
Qianqian Huang $^{1 \#}$, Kai Huang ${ }^{2 \#}$, Jinjun $\mathrm{Xue}^{3}$ \\ ${ }^{1}$ Operating Theatre, Tongde Hospital of Zhejiang Province, Hangzhou, China; ${ }^{2}$ Department of Orthopedics, Tongde Hospital of Zhejiang Province, \\ Hangzhou, China; ${ }^{3}$ Department of Pediatrics, Tongde Hospital of Zhejiang Province, Hangzhou, China \\ Contributions: (I) Conception and design: J Xue; (II) Administrative support: All authors; (III) Provision of study materials or patients: J Xue; (IV) \\ Collection and assembly of data: Q Huang; (V) Data analysis and interpretation: K Huang; (VI) Manuscript writing: All authors; (VII) Final approval \\ of manuscript: All authors. \\ "These authors contributed equally to this work and should be considered as co-first authors. \\ Correspondence to: Jinjun Xue. Department of Pediatrics, Tongde Hospital of Zhejiang Province, 234 Gucui Road, Hangzhou 310012 , China. \\ Email: xuejinjunzjtd@163.com.
}

\begin{abstract}
Background: To investigate the clinical experience and discuss the use of vacuum sealing drainage (VSD) combined with free anterolateral femoral flap grafting to treat pediatric foot and ankle soft tissue traumas.

Methods: This was a retrospective analysis of 16 pediatric patients with foot and ankle soft tissue traumas treated with free anterolateral femoral flap grafting from January 2015 to January 2018. After successful debridement and VSD, the clinical efficacy of the procedure was evaluated by observing parameters such as the color, shape, and texture of the flap on the receptor site.

Results: All patients were followed up for 23-32 months after surgery. Of the 16 patients, six had a flat flap appearance, while the remaining ten patients required flap repair surgery 6-9 months after the procedure. Eight of these ten patients had a satisfactory repair, and the remaining two patients had further repair surgery 9-10 months after the flap grafting. The postoperative results of all patients were satisfactory; no local infection, good flap survival, soft texture, glossy elasticity, and flat appearance. Statistical results showed only one case of local necrosis at the distal end of the flap, and the wound healed at stage II after redebridement and dressing change.

Conclusions: VSD combined with free anterolateral femoral flap grafting is an easy and reliable operation with a good prognosis and excellent clinical utility and feasibility for treating pediatric patients with foot and ankle soft tissue traumas.
\end{abstract}

Keywords: Vacuum sealing drainage (VSD); anterolateral femoral flap grafting; pediatric; soft tissue trauma

Submitted Aug 03, 2021. Accepted for publication Oct 19, 2021.

doi: $10.21037 /$ tp-21-399

View this article at: https://dx.doi.org/10.21037/tp-21-399

\section{Introduction}

With increased industrialization and mechanization, the incidence of large skin wounds and soft tissue traumas to the foot and ankle has grown. Fortunately, the development in microsurgery techniques has provided significant advances in the clinical treatment of such complex injuries (1). At present, free anterolateral femoral flap grafting is widely and effectively applied to treat adult patients with complex skin traumas and soft tissue damage. However, the same grafting procedure on pediatric patients is technically demanding and has a higher surgical risk due to the greater fragility and sensitivity of the pediatric body. In fact, there are few reports of pediatric patients that have undergone anterolateral femoral flap grafting (2). Severe open pediatric 
Table 1 General patient characteristics

\begin{tabular}{lc}
\hline Characteristics & Cases (\%) \\
\hline Age (years) & $6(37.5)$ \\
$>7$ & $10(62.5)$ \\
$<7$ & \\
Gender & $12(75.0)$ \\
Male & $4(25.0)$ \\
Female & \\
Foot & $9(56.25)$ \\
Right & $7(43.75)$ \\
Left & \\
\hline
\end{tabular}

injuries of the foot and ankle result in the exposure of bone and tendon and the loss of large portions of soft tissue, making them difficult to repair with skin grafts or adjacent flaps (3). To counter this problem, anterolateral femoral flap grafting is an ideal way to repair soft tissue damage in pediatric patients. From January 2015 to January 2018, 16 pediatric cases of soft tissue foot and ankle traumas were repaired by VSD in combination with free anterolateral femoral flap grafting. The procedure achieved good results, which are summarized below. We present the following article in accordance with the STROBE reporting checklist (available at https://dx.doi.org/10.21037/tp-21-399).

\section{Methods}

\section{Clinical information}

A total of 16 pediatric cases (age range, 5-11 years; average age $=7.8$ years) with soft tissue foot and ankle traumas were treated (informed consent was taken from all the patients). Twelve children were male, and four were female. There were nine cases of right foot injury and seven cases of left foot injury (Table 1). Thirteen injuries were caused by car accidents, and three were due to crushing injuries from heavy objects. The soft tissue damage was mainly in the dorsum of the foot and around the ankle joint, with areas of $7 \mathrm{~cm} \times 4 \mathrm{~cm}$ and $10 \mathrm{~cm} \times 5 \mathrm{~cm}$, respectively. All cases resulted in bone or tendon exposure, including seven metatarsal fractures and two tendon injuries. In view of the varying degrees of wound contamination, the wound was thoroughly debrided after admission and treated with vacuum sealing drainage (VSD) for about a week. The treatment of choice for all cases was elective grafting, with the operation taking place 6-12 days after admission. In addition, the size of the surgical excision of the flap area ranged from $10 \mathrm{~cm} \times 7 \mathrm{~cm}$ to $14 \mathrm{~cm} \times 8 \mathrm{~cm}$. All procedures performed in this study involving human participants were in accordance with the Declaration of Helsinki (as revised in 2013). The study was approved by Ethics Committee of Tongde Hospital of Zhejiang Province.

\section{Statistical analysis}

Using SPSS21.0 statistical software, all data were tested for normality. Mean SD was used to express normal distribution, and median (25 quantile, 75 quantile) was used to express skewed distribution data.

\section{Surgery method}

\section{VSD}

According to the routine laboratory procedure employed in our hospital, bacterial culture and drug sensitivity tests of the wound were performed following admission. Antibiotics were prescribed based on the drug sensitivity test results, followed by thorough debridement of the contaminated wound using the "carpet" method, in which all contaminated and necrotic tissues were removed, and the wound was washed with at least $6,000 \mathrm{~mL}$ of saline. Subsequently, Kirschner wires were used to repair the patient's injured tendon and metatarsal fracture. Appropriate VSD material was chosen to cover the wound completely based on the size and specific shape of the foot and ankle injury and without leaving any physiological dead spaces. A film was used to close the wound, followed by continuous vacuum drainage at $200 \mathrm{mmHg}$ pressure. A week later, the VSD material was removed. The flap repair was performed if the exposed tendon/bone showed no necrosis, the wound granulation tissue seemed clean, fresh and rubicund, and bled easily upon touching. If this was not the case, the debridement was repeated, the VSD material was replaced, and drainage was continued until the wound was fresh. After primary debridement and VSD, 14 patients showed controlled infection with fresh and rubicund surface granulation. The remaining two patients underwent secondary debridement and drainage to achieve a satisfactory result. The donor site of the anterolateral femoral flap was cleaned afterward.

\section{Flap design, excision, and grafting}

Two consecutive surgeries were performed that involved 
flap design, excision, closure of the donor site, the debridement of the receptor site, vascular dissection, vascular anastomosis, and flap suturing. The patient was positioned horizontally to excise the contralateral flap. Based on the basic principles of "point, line, and surface," initial determination of the peripheral projection of the descending branch of the lateral circumflex femoral artery (LCFA) was done from the anatomical position. Generally, the location was near the midpoint of the line from the anterior superior iliac spine to the external superior patellar border and the midpoint of the inguinal ligament. Subsequently, an ultrasonic Doppler blood flow detector was used to observe the specific skin penetration of the descending branch of the LCFA. The size, shape, depth of the wound, and the degree of pediatric fatness were taken into account when designing the flap. The donor site was marked schematically, and a flap slightly larger than the wound-generally greater than about $10 \%$ of the defected area-was used to facilitate tension-free suturing of the flap.

Tracheal intubation was used to administer anesthesia to the patient. Subsequently, starting from the medial side of the flap along the schematic markings, the skin, subcutaneous tissue, and deep fascia were incised layer by layer to expose the muscle tissue. Then, the deep fascia was intermittently sutured with the flap for a few stitches to prevent separation of the fascia from the skin. Blunt dissection along the gap between the rectus femoris and vastus lateralis muscle was performed to explore the unexposed and carefully protected descending branch of the LCFA. The larger branches emanating laterally from the descending branch of the LCFA were sought out and dissected from above to below, followed by the complete dissection of the superior, inferior, and medial edges of the flap. The retrograde tracing operation was performed after carefully separating the completely exposed perforating branch with its vastus lateralis muscle. Part of the perforating branch was gradually severed until it was completely exposed and had clear continuity with the descending branch. At the end of the procedure, the lateral edge of the flap was incised, followed by dissection of the free descending trunk and ligation of the distal vessel pedicle. Additionally, the pedicle division was performed according to the length of the desired vessel pedicle in the receptor site, resulting in the formation of an anterolateral femoral flap pedicled with the descending branch of the LCFA.

Once the pedicle division was placed over the receptor site, repeated wound and anterolateral femoral flap rinsing was performed. Several temporary sutures were made and fixed, and a microscope was used to select either the posterior tibial artery and vein or the anterior tibial artery and vein for anastomosis. In this group, 14 anastomoses targeted the anterior tibial artery and vein, while the remaining two anastomoses targeted the posterior tibial artery and vein. Several drainage strips were placed after the flap was tension-free sutured to the circumferential skin of the wound. Meanwhile, the donor site was usually closed by direct-suturing with free skin-grafting covering the inadequate area.

\section{Postoperative management and follow-up}

The flap blood circulation was kept under close observation after surgery, and the surgery site was kept warm with a heat lamp for 1 week, along with conventional anti-infective, anti-spasmodic, and anticoagulant drug administration. The use of intravenous analgesic pumps caused vascular crisis due to severe pain in three patients post-surgery, which was relieved by hibernation therapy. Symptomatic drug treatment was not helpful for pain relief in one patient who had developed a vascular crisis, and for that case, emergency exploratory surgery was performed that revealed venous reflux obstruction. After reanastomosis of the vein, most of the flap survived, with necrosis in the distal $1 / 5$ of the flap, and the wound healed after two weeks of debridement and dressing changes. All cases were followed up for 23-32 months. Six patients showed satisfactory results with a flat flap appearance that did not affect daily activities, such as walking in shoes. The remaining ten patients needed flap repair surgery 6-9 months after grafting due to the swollen appearance of the flap. Eight of these patients required one repair, and the remaining two patients underwent a second flap repair 9-10 months after the initial graft. Postoperative wound analysis included observation of healing in the receptor site; the shape, color, and texture of the flap; the donor site scar, and the recovery of functional activities.

\section{Two typical cases}

Typical case 1 is a 5 -year-old female patient with dorsal skin soft tissue necrosis of the right foot (Figure 1). The wound condition at admission is shown in Figure 1A. After admission, we initially performed debridement and VSD negative pressure aspiration. The wound appearance one week after operation is shown in Figure 1B. Figure $1 C$ shows the wound condition after the free anterolateral femoral flap grafting repair. The postoperative condition after the flap repair can be seen in Figure $1 F$, and the flap condition is shown one week after surgery (Figure 1D), 8 months 

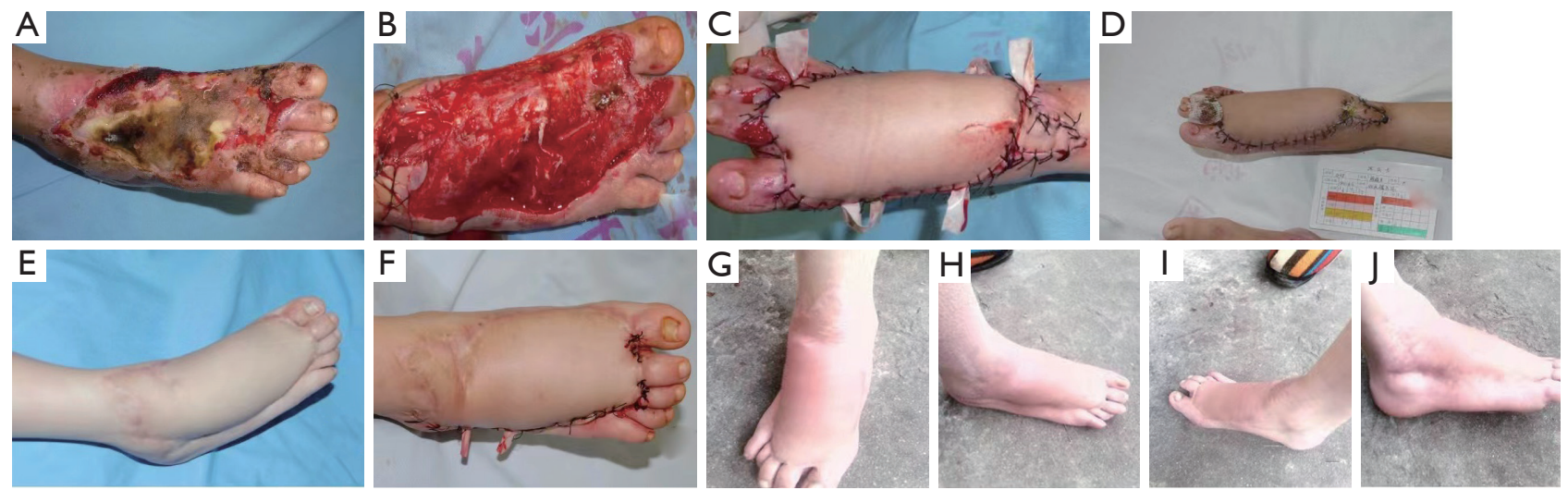

Figure 1 Typical case 1. A 5-year-old female patient with dorsal skin soft tissue necrosis of the right foot. (A) Wound condition at admission; (B) wound condition one week after debridement and VSD; (C) condition after free anterolateral femoral flap grafting repair; (D) flap condition one week after surgery; (E) flap condition 8 months after surgery; (F) postoperative condition after flap repair; (G-J) flap condition 48 months after surgery. VSD, vacuum sealing drainage.
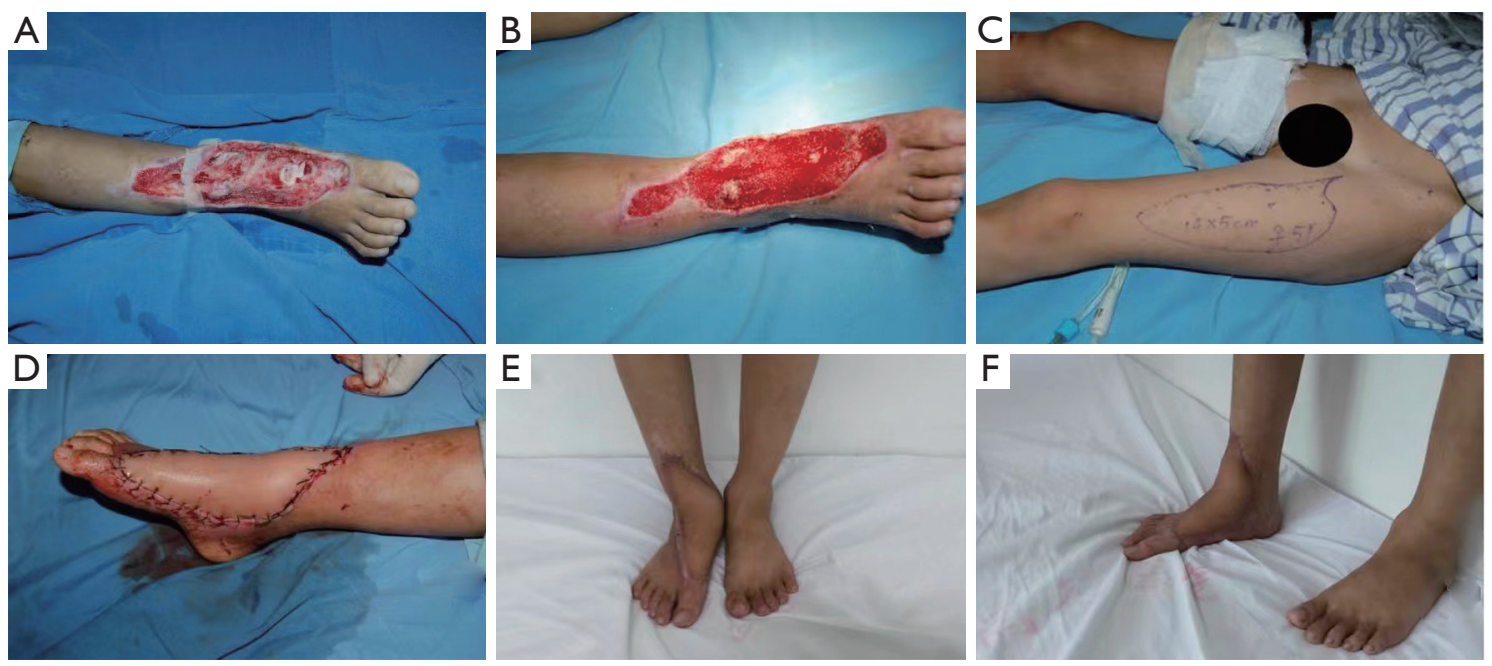

Figure 2 Typical case 2. The patient was a 5 -year-old female with a dorsal skin and soft tissue trauma to the right foot with tendon and bone exposure. (A) Wound condition at admission; (B) wound condition one week after debridement and VSD; (C) design of free anterolateral femoral flap; (D) postoperative condition of free anterolateral femoral flap grafting repair; (E,F) flap condition 18 months after surgery. VSD, vacuum sealing drainage.

after surgery (Figure $1 E$ ), and 48 months after surgery (Figure 1G-1f). Typical case 2 is a female, aged 5 years, with a dorsal skin and soft tissue defect of the right foot with tendon and bone exposure (Figure 2). The wound condition at admission and one week after debridement and VSD are shown in Figure $2 A$ and Figure 2B, respectively. The free anterolateral femoral flap design can be seen in Figure $2 C$, the postoperative condition of the free anterolateral femoral flap grafting repair is shown in Figure $2 D$, and the flap condition 18 months after surgery is shown in Figure $2 E$ and Figure $2 F$.

\section{Results}

All 16 patients in this retrospective analysis had successful surgeries without any intraoperative complications. Three patients experienced postoperative vascular crises due to severe pain, and hibernation therapy was used to 
relieve the pain after consultation with a pediatrician. One patient experienced a vascular crisis that could not be relieved by symptomatic drug treatment, so emergency exploratory surgery was performed that revealed a venous reflux obstruction. The majority of the flap survived after reanastomosis of the vein. The rest of the patients showed no adverse reactions post-surgery with good vitals and mental state. In stage I, all donor sites were infection-free and healed, and the flap in the receptor site survived well. There was only one case of local necrosis at the distal end of the flap, which healed in stage II after redebridement and dressing change. All patients were kept under observation 4-36 months after surgery, and ten patients required flap repair. The final feedback results were as follows: A flat flap appearance with elasticity and texture similar to the surrounding normal skin, a small donor site scar, recovery of functional activity of the affected limb, no local pain, loss of sensation or functional impairment, and the patient had no difficulty walking in shoes. Overall, the patients were satisfied with the postoperative condition of their limb.

\section{Discussion}

\section{Advantages of the VSD technique in pediatric trauma}

Since its first creation in 1993 by Fleischman (4) at ULM University in Germany, the VSD technique has been widely used to treat severe traumatic wounds. It has the advantages of rapidly reducing periwound edema and promoting the rapid growth of granulation tissue and has achieved conspicuous results. The main points of VSD are: (I) the treatment time is obviously shortened, the patient may avoid the pain which changes the medicine; (II) VSD is carried out in a closed environment to effectively prevent cross-infection; (III) continuous negative pressure suction promotes the fluid flow from the wound tissue to the drainage tube, which provides an effective, continuous and auxiliary power to the wound circulation; (IV) for the superficial wound, can play close to the tissue, reduce the wound surface, reduce the skin graft area effect.

However, unlike adults, children have delicate tissues and fragile tendons, so they suffer from injuries that can easily lead to large avulsions with skin and soft tissue damage. In the worst cases, bones, joints, tendons, blood vessels, and nerves may all get injured simultaneously, making the wound a complex and serious one that creates great difficulty for its clinical treatment (5). Considering the poor resistance of pediatric patients, treating their soft tissue limb wounds with VSD draws necrotic materials and their secretions out of the body more efficiently in a shorter time. VSD can also protect the wound from harmful airborne microorganisms, reduce the infection probability, and shorten the cycle of antibiotic use (6). Many studies support the use of VSD for rapid and effective promotion of wound healing. Generally speaking, for thoroughly debrided wounds, fresh, granular, red tissue can be seen 4-5 days after VSD (7). Because the pediatric population has a low pathophysiological pain tolerance, the use of VSD helps children avoid the pain caused by long-term dressing changes and debridement. The procedure can also reduce the workload of medical and nursing staff and shorten the course of treatment (8). The use of VSD after first-stage debridement not only facilitates quick, simple, and effective treatment of the wound but also reduces the traumatic effect on the child, decreases the chance of infection, and reduces complications. As such, VSD provides an optimal environment that guarantees successful subsequent free flap grafting (9).

\section{Characteristics and precautions of pediatric lateral femoral free flap}

The anterolateral femoral flap is a thigh flap with the descending branch of the LCFA as the vascular pedicle. The advantages of the LCFA include a long vascular pedicle, thick caliber, concealed location, and a low impact on the donor site. Known as the "universal flap," it has been widely applied in the repair of soft tissue skin damage since its first proposal in 1983, when it was perfected by anatomical studies conducted by Chinese scholars (10). However, there are additional requirements for pediatric lateral femoral free flap grafting. Firstly, pediatric patients are in the growth and development stage of life, so the impact of the scar on their future quality of life requires serious consideration. Secondly, the small caliber and relatively weak walls of pediatric vessels make intraoperative stimulation prone to vasospasm, which affects the efficacy of surgical operations (11). Therefore, it is essential to master and perform the entire vascular anatomy and anastomosis procedure with precision and care. Furthermore, pediatric patients have more difficulty understanding the meaning of surgery than adults, and their lower tolerance to anesthesia, pain, surgical trauma, blood loss, and resistance to infection should be considered; adequate preoperative preparation is essential in this regard. Emotionally, detailed explanations and clear communication with pediatric patients and their 
parents are needed to obtain full cooperation. Technically, Color Doppler Ultrasound (CDS) combined with Multislice Dimensional CT Angiography (MDCTA) can be used to detect and accurately locate the position of the perforating branch (12) and mark it precisely. For clarification of the division of labor and for tracking surgery speed and time, it is essential to closely monitor and maintain blood volume stability intraoperatively. In this way, surgery time and the chances of arterial spasm can be reduced, and the safety and success rate of surgery be improved (13). In addition, careful postoperative supervision is required to prevent other complications. Also, given the immaturity of the pediatric vascular system with its thin diameter and poor pressure capacity, it is of great importance to anastomose as many large veins as possible while avoiding medically derived extravascular membrane injury so as to promote venous reflux and prevent the occurrence of venous crisis due to injury or edema (14). The main complications after surgery are infection, ISCHEMIA and necrosis of skin flap, hematoma under skin flap, joint stiffness and so on. If the skin flap becomes infected after the operation, we can take the following measures: strengthen the dressing change, pay attention to the aseptic operation, look for the pathogenic bacteria, choose oral or intravenous antibiotic treatment according to the result of drug sensitivity.

In summary, comprehensive preoperative preparation, active communication, delicate intraoperative operation, clear division of labor, close postoperative observation, and meticulous care are essential to ensure a successful surgery.

\section{Summary and prospects}

In summary, Anterolateral thigh flap transplantation is a good method to repair soft tissue injury in adults and children. And the main steps of staged surgical treatment: (I) thoroughly debride, repair tendon injury; (II) continuous VSD negative pressure suction; (III) skin flap design, cut, close wound; (IV) debridement, vascular anatomy, vascular anastomosis and flap suture of the recipient area. The 16 pediatric patients included in this study underwent VSD in the first stage of active and thorough debridement, while keeping pediatric characteristics under consideration, which laid a solid ground for a good soft tissue bed for later grafting. In stage II, free anterolateral femoral flap grafting was used to repair the soft tissue damage to the foot and ankle, where satisfactory results were achieved with active postoperative complication prevention and meticulous care. VSD combined with free anterolateral femoral flap grafting is beneficial in many ways, including a concealed donor site, smaller scar, anatomical constancy, high flap viability (15), shorter course, faster wound healing, and a reduced infection rate (16). Therefore, these advantages make this technique the method of choice for repairing pediatric foot and ankle soft tissue traumas with an easy operation, reliable efficacy, and a good prognosis with significant clinical value. Given the specific characteristics of the pediatric patient, the procedure and operating technique is challenging for the surgeon as it requires high patience, concentration, and care. In addition, because of the limitations of the free anterolateral femoral flap itself, further research is needed to improve the quality of the procedure and reduce complications.

\section{Conclusions}

VSD combined with free anterolateral thigh flap is a simple and effective method for the treatment of traumatic soft tissue defect of foot and Ankle in children.

\section{Acknowledgments}

Funding: This study was funded by the Zhejiang Province Public Welfare Technology Application Research Project (LGF21H270004), the Natural Science Foundation of Zhejiang Province (LY20H270002), the Medical Science and Technology Project of Zhejiang Province (2020RC048, 2021KY600), and the Chinese Medicine Research Program of Zhejiang Province (2020ZQ006, 2021ZB060). The funders had no role in the study design, data collection and analysis, decision to publish, or manuscript preparation.

\section{Footnote}

Reporting Checklist: The authors have completed the STROBE reporting checklist. Available at https://dx.doi. org/10.21037/tp-21-399

Data Sharing Statement: Available at https://dx.doi. org/10.21037/tp-21-399

Conflicts of Interest: All authors have completed the ICMJE uniform disclosure form (available at https://dx.doi. org/10.21037/tp-21-399). The authors have no conflicts of 
interest to declare.

Ethical Statement: The authors are accountable for all aspects of the work in ensuring that questions related to the accuracy or integrity of any part of the work are appropriately investigated and resolved. All procedures performed in this study involving human participants were in accordance with the Declaration of Helsinki (as revised in 2013). The study was approved by Ethics Committee of Tongde Hospital of Zhejiang Province. Informed consent was taken from the patients' parents or legal guardians.

Open Access Statement: This is an Open Access article distributed in accordance with the Creative Commons Attribution-NonCommercial-NoDerivs 4.0 International License (CC BY-NC-ND 4.0), which permits the noncommercial replication and distribution of the article with the strict proviso that no changes or edits are made and the original work is properly cited (including links to both the formal publication through the relevant DOI and the license). See: https://creativecommons.org/licenses/by-nc-nd/4.0/.

\section{References}

1. Zocchi ML. Regenerative assisted microsurgery (RAM) and regenerative assisted supermicrosurgery (RASM): the future of microsurgery? Eur J Plast Surg 2021. [Epub ahead of print]. doi: 10.1007/s00238-021-01812-6.

2. Xu ZF, Duan WY, Tan XX, et al. Reconstruction of Complex Total Parotidectomy Defect With a Chimeric Anterolateral Thigh Perforator Flap and Vascularized Motor Branch of Femoral Nerve Grafting. J Oral Maxillofac Surg 2015;73:2448.e1-7.

3. Xia CD, Di HP, Xue JD, et al. Effects of free anterolateral femoral or medial calf flaps in the repair of severe facial burns. Zhonghua Shao Shang Za Zhi 2019;35:512-6.

4. Fleischmann W, Strecker W, Bombelli M, et al. Vacuum sealing as treatment of soft tissue damage in open fractures. Unfallchirurg 1993;96:488-92.

5. Meentken Maya G, van der Ende Jan, del Canho Riwka, et al. Psychological outcomes after pediatric hospitalization: the role of trauma type. Children's Health Care 2021;50:278-92.

6. Sui X, Cao Z, Pang X, et al. Reconstruction of moderatesized soft tissue defects in foot and ankle in children: Free deep inferior epigastric artery perforator flap versus circumflex scapular artery perforator flap. J Plast Reconstr Aesthet Surg 2019;72:1494-502.

7. Hao Z. Application of Pelnac ${ }^{\circledR}$ Artificial Dermis
Combined with VSD in the Repair of Limb Wounds. J Invest Surg 2020;33:642-3.

8. Bergmann M, Germann CP, Nordmeyer J, et al. Shortand Long-term Outcome After Interventional VSD Closure: A Single-Center Experience in Pediatric and Adult Patients. Pediatr Cardiol 2021;42:78-88.

9. Li RG, Ren GH, Tan XJ, et al. Free flap transplantation combined with skin grafting and vacuum sealing drainage for repair of circumferential or sub-circumferential soft-tissue wounds of the lower leg. Med Sci Monit 2013;19:510-7.

10. Walczak DA, Grajek M, Migacz E, et al. Preoperative tracing of lateral femoral cutaneous nerve with sonography for sensory anterolateral thigh free flap reconstruction. J Reconstr Microsurg 2020;36:e3-e4.

11. Bhogesha S, Rimal D, Song C. The descending branch of lateral circumflex femoral artery (LCFA) as recipient pedicle for free flap cover of complex defects around the knee. Microsurgery 2019;39:573-4.

12. Mughal M, Gabuniya N, Zoccali G, et al. Functional Outcomes of the Medial Sural Artery Perforator Flap in Oral Cavity Reconstruction. Ann Plast Surg 2020;85:256-9.

13. Silva AK, Portugal L, Blair EA. In Reference to The Medial Sural Artery Perforator Flap: A Better Option in Complex Head and Neck Reconstruction? Laryngoscope 2020;130:E456.

14. Sue GR, Kao HK, Borrelli MR, et al. The versatile free medial sural artery perforator flap: An institutional experience for reconstruction of the head and neck, upper and lower extremities. Microsurgery 2020;40:427-33.

15. Kurlander DE, Wu-Fienberg Y, Long TC, et al. The Anterolateral Thigh-Medial Femoral Condyle Chimeric Flow-through Flap for Posttraumatic Wrist Arthrodesis. Tech Hand Up Extrem Surg 2019;23:143-5.

16. He Y, Jin SF, Zhang ZY, et al. A prospective study of medial sural artery perforator flap with computed tomographic angiography-aided design in tongue reconstruction. J Oral Maxillofac Surg 2014;72:2351-65.

(English Language Editor: D. Fitzgerald)

Cite this article as: Huang Q, Huang K, Xue J. Vacuum sealing drainage combined with free anterolateral femoral skin flap grafting in 16 cases of pediatric soft tissue damage to the foot and ankle. Transl Pediatr 2021;10(10):2489-2495. doi: 10.21037/tp-21-399 